\title{
DUS Characterization of Elite Improved Lines of Greengram [Vigna radiata (L.) Wilczek]
}

\author{
A. Sheena Sabatina, M. Lal Ahamed", J. V. Ramana and N. Harisatyanarayana \\ Department of Molecular Biology and Biotechnology, Advanced Post Graduate Centre, \\ Lam, Guntur, India \\ *Corresponding author
}

\section{A B S T R A C T}

\section{Keywords}

Distinctness,

Uniformity and

Stability (DUS),

Greengram,

Identification and

protection,

Morphological

characterization

Article Info

Accepted:

20 December 2020

Available Online:

10 January 2021
The study was carried out to characterize elite improved lines of Greengram genotypes developed at Regional Agricultural Research Station (RARS), Lam, Guntur using DUS descriptors. The 29 elite improved lines and one released variety (LGG 460) were evaluated and characterized for 24 DUS descriptors i.e., anthocyanin colouration during cotyledonary stage, plant, stem, leaf, flower, pod and seed characters. 14characters out of 24 characters of DUS descriptors differed significantly indicating a large and exploitable amount of genetic variability for the individual elite improved line profile development for identification and protection. The elite lines are similar for the important plant traits like semi erect and determinate growth habit but the development of erect types is the need of hour and indicates the incorporation of new germplasm for the improvement of this trait in the present material. The DUS descriptor data generated with unique profiles of the elite improved lines can be used for the registration with PPV \& FRA and seed purity testing.

\section{Introduction}

Greengram [Vigna radiata (L.) Wilczek] $(2 n=2 x=22)$, a self-pollinated crop of 579 $\mathrm{Mb} / 1 \mathrm{Cgenome}$ size, is native to Indian subcontinent. It fixes atmospheric nitrogen in the soils like other pulse crops. It forms a major source of protein $(240 \mathrm{~g} / \mathrm{kg})$ and a range of micronutrients to the diet of vegetarian population of India (Ramakrishnan et al., 2013). The easily digested proteins and carbohydrates of greengram are low in phytic acid, which reduces iron and zinc bioavailability after consumption. In 2018-19, the area and production of greengram in India are $4.25 \mathrm{~m}$ ha and 2.41 million tones, respectively (Anonymous, 2019). This short duration crop is amenable to diverse environmental situation including multiple cropping systems to expand its area and production and making India a nutritionally secure place for its growing population. The greengram production still depends on the small to medium varieties, which reach 
maturity in 60-80 days. The major problems in greengram production are indeterminate growth types which need to be harvested multiple times; susceptibility to diseases and insect pests, and pod shattering. The average yields of the released varieties from various parts of the country are around 600kgs/ ha and this is far below the world average and is attributed to narrow genetic base and absence of suitable genotypes for different cropping situations. Further, it also highlighted the importance of development of new lines for yield to meet the growing needs of the nation (Abbas et al., 2010; Pratap et al., 2018).

Characterization of germplasm is the basic step in forming groups of lines having similar characteristics to get an idea of variability present in the lines and their utilization in breeding programmes (Lee et al., 2004; Piyada et al., 2010). Normally, agromorphological traits are used for the identification of lines as they are seen easily with naked eyes during physical purity maintenance.

Characterization and identification of new cultivars is essential for their efficient utilization and conservation. Normal morphological descriptions of lines by the plant breeders are inadequate to get the complete picture of the lines and highlight the need of standard procedures described by a competent authority like PPV \& FRA (Protection of Plant Varieties and Farmers' Rights Authority) to characterize the genotypes. Thus, characterization using Distinctness, Uniformity and Stability (DUS) is of great significance in the era intellectual property rights for the protection of lines as well as quality seed production and certification (Janghel et al., 2020). These descriptors are simple, cheap and do not require any sophisticated laboratory techniques. Therefore, characterization and evaluation of variation in the elite improved lines of greengram would be of great significance in overall improvement of qualitative and quantitative characteristics and protection. Katiyar et al., (2007) explained the genetic relationships among the breeding lines of greengram present in India using morphological characters. Keeping these points in view, this work is planned to study the DUS characteristics of the elite improved lines of greengram of Regional Agricultural Research Station (RARS), Lam, Guntur, Andhra Pradesh, India.

\section{Materials and Methods}

The experimental material consisted of 29 elite improved lines of greengram and a released variety (LGG 460) developed at Regional Agricultural Research Station (RARS), Lam, Guntur, Andhra Pradesh. The experiment was conducted in a randomized block design with three replications during Rabi 2019-2020 at Regional Agricultural Research Station, Lam, Guntur. All the lines were planted in four rows of 4 meters length adopting 40x10 $\mathrm{cm}$ spacing among the lines and plants, respectively for the morphological characterization. The observations were recorded at specified stages of crop growth period when the traits under study had full expression i.e., different growth stages. The anthocyanin pigmentation at cotyledonary stage was observed at unfolded stage (5-10 days after sowing). The characters, growth and plant habits, stem and leaf characters were noted at days to $50 \%$ flowering stage. The characters, plant height and premature pod colour were observed in fully developed pod. Pod characters like colour, curvature, position and length were recorded at maturity stage of the pod. Seed traits like colour, coat luster, shape, size were characterized after the harvest of the crop. The morphological traits observation was done as per the DUS guidelines suggested by the Protection of Plant Varieties \& Farmers' Rights Authority (2007). 


\section{Results and Discussion}

Characterization of thirty elite improved lines of greengram was carried out using DUS descriptors. The characters of anthocyanin colouration during cotyledonary stage, plant characteristics, stem, leaf, flower, pod and seed characteristics during different growth stages of crop growth differed significantly for 14 characters out of 24 characters of DUS descriptors indicating a large and exploitable amount of genetic variability for the individual elite improved line profile development for identification and protection (Table -1). Katiyar et al., (2008) also exploited DUS characterization in greengram for the identification and protection.

Anthocyanin colouration is normally considered as important morphological marker in greengram to discriminate the lines into two groups based on their presence or absence and was recorded at cotyledonary stage (Mukherjee and Pradhan, 2002; Khattak et al., 2000). In the present investigation, eleven genotypes were without pigmentation and nineteen genotypes showed anthocyanin colour indicating the existence of variation among the lines and to use as selection criteria for identification of the lines (Table2).

\section{Plant characters}

Plant morphological characters like petiole colour, habit, growth habit and height were observed at days to 50 per cent flowering and at fully developed green pod stages of plant, respectively, and can be used as criteriain varietal purity maintenance and identification. All the genotypes showed green petiole colour with purple splashes. Further, all genotypes were grouped into semi- erect plant growth habit and determinate plant type suggesting they are highly similar for these traits. Normally, erect plant types are preferred as they get very good sun shine and efficiently synthesize food for the growth and development of plant. Thus, there is a need to incorporate this trait in RARS, Lam, Guntur germplasm for future new lines development with this trait. The genotypes were grouped into short $(<50 \mathrm{~cm})$ with twenty eight genotypes and medium $(50-70 \mathrm{~cm})$ with two genotypes i.e., LGG695 and LGG696 based on plant height. The plant height was maximum in the genotype, LGG 696 $(51.50 \mathrm{~cm})$ and was the lowest in LGG 687 $(33.87 \mathrm{~cm})$. Sunil et al., (2013) reported the presence of great variability for this traits in their study using 89 accessions from Andhra Pradesh.

\section{Stem characters}

Stem morphological characters, colour and pubescence, were recorded at days to 50\% flowering stage of plant. All the genotypes showed green stem colour with purple splashes and pubescence indicating all the genotypes are similar for these traits and not useful in identification and purity maintenance.

\section{Leaf characters}

Leaf morphological characters like leaflet lobes, shape, colour, size and vein colour were observed at days to 50 per cent flowering stage of the plant. These characters play an important role in the yielding ability of the genotypes, as the leaves are the points of food synthesis and transpiration site of the plants. All these characters except leaf vein colour, showed variability and the genotypes were categorized into distinct groups (Table2).

The leaflet was lobed in four genotypes, whereas, it was absent in remaining twenty six genotypes. Leaf shape was deltoid in eleven genotypes; ovate in fourteen genotypes 
and lanceolate in five genotypes. Leaf colour was green in nine genotypes and dark green in remaining twenty one genotypes. Leaf size was small in two genotypes and medium in twenty eight genotypes. Leaf vein colour was greenish purple in all the genotypes. The leaf traits (foliage colour, leaf shape, leaflet lobes and leaf size) showed very good variation and are useful in the characterization but the effect of environmental factors is very high on these traits as they are polygenically controlled.

\section{Flower characters}

Flower characters viz., colour and time of flower, were observed at days to $50 \%$ flowering stage of plant. Flower colour is reliable morphological marker and distinguishing the greengram genotypes but all the thirty genotypes showed the similar flower petal colour i.e., yellow (Table-2). Hence, this trait is of no use in discriminating the genotypes in the present material. Based on time of flowering, the genotypes were categorized into early (< 40 days) with nineteen genotypes and medium (40-50 days) with eleven genotypes.

Flowering time varied with genotypes ranging from 33 days (LGG 691) to 48 days (LGG 698). Therefore, these lines hold great promise as early maturing genotypes in greengram. The short duration genotypes can overcome the adverse effects of terminal heat stress and untimely rains at the time of harvest. These genotypes are helpful in expanding the area under greengram during summer season (Pratap et al., 2013).Jain et al., (2002) reported the usefulness of flower characteristics in characterization of greengram germplasm.

\section{Pod characters}

Greengram pod characteristics are highly useful in identification of the genotypes. The pod characters like colour of premature pod, pod pubescence and pod position, were observed at fully developed green pod stage while pod colour, curvature of mature pod and pod length were noted during plant maturity stage before harvest (Table-2). These characteristics influence the yielding ability of the plant and are considered as the main yield attributing traits. All these characters showed variation and were categorized into distinct groups except for the trait, pod colour.

The trait, colour of premature pod, was green in five genotypes and green with pigmented suture in remaining twenty five genotypes. Pod pubescence was absent in five genotypes and present in twenty five genotypes. Pod position was above canopy in twenty genotypes, intermediate in seven genotypes and not visible in three genotypes. Kaur et al., (2017) also reported similar trend for this trait in their study. Pod colour is a quite useful morphological marker and may be used in quality seed production programmes at maturity stage to monitor the mixture of other varieties but all the lines showed black pods at the time of maturity indicating this trait is of no use for identification of lines in the present material.

Curvature of mature pod was straight in twenty four genotypes and curved in six genotypes. Pod length was observed to have limited variability in the present study as twenty four genotypes recorded short pod length and six genotypes showed medium pod length. The longest pod was observed in LGG $700(8.77 \mathrm{~cm})$ and the lowest was seen in LGG $692(6.03 \mathrm{~cm})$. Thus, these pod characteristics can be exploited for identification and characterization but are found to be variable due to more number of genes and environmental influence on the expression. Sunil et al., (2014) observed straight pods without curvature in their study. 
Table.1 DUS descriptors of thirty Greengram elite improved lines developed at RARS, Lam, Guntur, Andhra Pradesh

\begin{tabular}{|c|c|c|c|c|c|c|c|c|c|c|c|c|c|c|c|c|c|c|c|c|c|c|c|c|c|}
\hline S.No & Genotypes & $\begin{array}{l}\text { Antho } \\
\text { Cyanin } \\
\text { colour } \\
\text { ation }\end{array}$ & $\begin{array}{c}\text { Time } \\
\text { of } \\
\text { flowerin } \\
\mathrm{g}\end{array}$ & $\begin{array}{c}\text { Plant } \\
\text { growth } \\
\text { habit }\end{array}$ & $\begin{array}{l}\text { Plant } \\
\text { habit }\end{array}$ & $\begin{array}{l}\text { Stem } \\
\text { colour }\end{array}$ & $\begin{array}{c}\text { Stem } \\
\text { pubesce } \\
\text { nce }\end{array}$ & $\begin{array}{l}\text { Leaflet } \\
\text { lobes }\end{array}$ & $\begin{array}{l}\text { Leaf } \\
\text { shape }\end{array}$ & $\begin{array}{l}\text { Leaf } \\
\text { colour }\end{array}$ & $\begin{array}{l}\text { Leaf } \\
\text { vein } \\
\text { colour }\end{array}$ & \begin{tabular}{|l|} 
Petiole \\
colour
\end{tabular} & $\begin{array}{l}\text { Leaf } \\
\text { size }\end{array}$ & $\begin{array}{l}\text { Flower } \\
\text { colour }\end{array}$ & $\begin{array}{l}\text { Colour } \\
\text { of } \\
\text { prematu } \\
\text { re pod }\end{array}$ & $\begin{array}{l}\text { Pod } \\
\text { Pubes } \\
\text { cence }\end{array}$ & $\begin{array}{c}\text { Pod } \\
\text { positio } \\
n\end{array}$ & $\begin{array}{l}\text { Plant } \\
\text { height }\end{array}$ & $\begin{array}{l}\text { Pod } \\
\text { colour }\end{array}$ & $\begin{array}{c}\text { Curvat } \\
\text { ure of } \\
\text { mature } \\
\text { pod }\end{array}$ & $\begin{array}{l}\text { Pod } \\
\text { length }\end{array}$ & $\begin{array}{l}\text { Seed } \\
\text { colour }\end{array}$ & $\begin{array}{l}\text { Seed } \\
\text { luster }\end{array}$ & $\begin{array}{c}\text { Seed } \\
\text { shape }\end{array}$ & $\begin{array}{l}\text { Seed } \\
\text { size }\end{array}$ \\
\hline 1. & LGG 684 & 1 & 5 & 5 & 1 & 2 & 9 & 9 & 1 & 2 & 2 & 2 & 5 & 3 & 1 & 9 & 1 & 3 & 2 & 1 & 3 & 3 & 1 & 1 & 5 \\
\hline 2. & LGG 685 & 9 & 5 & 5 & 1 & 2 & 9 & 1 & 2 & 2 & 2 & 2 & 7 & 3 & 1 & 9 & 2 & 3 & 2 & 1 & 3 & 2 & 1 & 1 & 5 \\
\hline 3. & LGG 686 & 9 & 3 & 5 & 1 & 2 & 9 & 1 & 1 & 2 & 2 & 2 & 7 & 3 & 2 & 9 & 1 & 3 & 2 & 1 & 3 & 3 & 1 & 1 & 5 \\
\hline 4. & LGG 687 & 1 & 3 & 5 & 1 & 2 & 9 & 1 & 1 & 2 & 2 & 2 & 7 & 3 & 1 & 9 & 1 & 3 & 2 & 1 & 3 & 2 & 1 & 1 & 5 \\
\hline 5. & LGG 688 & 9 & 3 & 5 & 1 & 2 & 9 & 1 & 3 & 1 & 2 & 2 & 7 & 3 & 2 & 9 & 1 & 3 & 2 & 1 & 3 & 2 & 2 & 2 & 5 \\
\hline 6. & LGG 689 & 1 & 3 & 5 & 1 & 2 & 9 & 1 & 2 & 2 & 2 & 2 & 7 & 3 & 1 & 9 & 2 & 3 & 2 & 1 & 3 & 2 & 2 & 1 & 5 \\
\hline 7. & LGG 690 & 1 & 3 & 5 & 1 & 2 & 9 & 1 & 2 & 2 & 2 & 2 & 7 & 3 & 1 & 9 & 1 & 3 & 2 & 1 & 5 & 2 & 1 & 1 & 5 \\
\hline 8. & LGG 691 & 9 & 3 & 5 & 1 & 2 & 9 & 1 & 1 & 2 & 2 & 2 & 5 & 3 & 2 & 1 & 1 & 3 & 2 & 1 & 3 & 2 & 1 & 2 & 5 \\
\hline 9. & LGG 692 & 9 & 5 & 5 & 1 & 2 & 9 & 1 & 2 & 2 & 2 & 2 & 5 & 3 & 2 & 1 & 1 & 3 & 2 & 1 & 3 & 2 & 2 & 2 & 5 \\
\hline 10. & LGG 693 & 9 & 5 & 5 & 1 & 2 & 9 & 1 & 1 & 2 & 2 & 2 & 7 & 3 & 2 & 9 & 2 & 3 & 2 & 1 & 3 & 2 & 1 & 2 & 5 \\
\hline 11. & LGG 694 & 9 & 3 & 5 & 1 & 2 & 9 & 1 & 2 & 2 & 2 & 2 & 7 & 3 & 2 & 1 & 1 & 3 & 2 & 1 & 3 & 2 & 1 & 1 & 5 \\
\hline 12. & LGG 695 & 9 & 3 & 5 & 1 & 2 & 9 & 1 & 2 & 1 & 2 & 2 & 7 & 3 & 2 & 9 & 1 & 3 & 2 & 1 & 3 & 2 & 1 & 1 & 5 \\
\hline 13. & LGG 696 & 9 & 3 & 5 & 1 & 2 & 9 & 1 & 2 & 2 & 2 & 2 & 5 & 3 & 2 & 9 & 1 & 3 & 2 & 3 & 3 & 2 & 1 & 1 & 5 \\
\hline 14. & LGG 697 & 9 & 5 & 5 & 1 & 2 & 9 & 1 & 2 & 2 & 2 & 2 & 5 & 3 & 2 & 1 & 3 & 3 & 2 & 3 & 3 & 2 & 2 & 2 & 5 \\
\hline 15. & LGG 698 & 1 & 5 & 5 & 1 & 2 & 9 & 9 & 2 & 2 & 2 & 2 & 5 & 3 & 2 & 9 & 1 & 3 & 2 & 1 & 5 & 2 & 1 & 2 & 5 \\
\hline 16. & LGG 699 & 9 & 5 & 5 & 1 & 2 & 9 & 1 & 1 & 2 & 2 & 2 & 7 & 3 & 2 & 9 & 3 & 3 & 2 & 3 & 3 & 2 & 2 & 2 & 5 \\
\hline 17. & LGG 700 & 1 & 3 & 5 & 1 & 2 & 9 & 1 & 1 & 1 & 2 & 2 & 7 & 3 & 2 & 9 & 1 & 3 & 2 & 1 & 5 & 3 & 1 & 2 & 5 \\
\hline 18. & LGG 701 & 1 & 3 & 5 & 1 & 2 & 9 & 9 & 3 & 2 & 2 & 2 & 5 & 3 & 2 & 9 & 1 & 3 & 2 & 1 & 3 & 2 & 2 & 2 & 5 \\
\hline 19. & LGG 702 & 1 & 5 & 5 & 1 & 2 & 9 & 1 & 2 & 2 & 2 & 2 & 5 & 3 & 2 & 9 & 1 & 3 & 2 & 3 & 5 & 2 & 1 & 1 & 5 \\
\hline 20. & LGG 703 & 1 & 3 & 5 & 1 & 2 & 9 & 1 & 2 & 1 & 2 & 2 & 5 & 3 & 2 & 9 & 3 & 3 & 2 & 1 & 3 & 2 & 1 & 1 & 5 \\
\hline 21. & LGG 704 & 9 & 3 & 5 & 1 & 2 & 9 & 1 & 3 & 1 & 2 & 2 & 5 & 3 & 2 & 9 & 2 & 3 & 2 & 1 & 5 & 2 & 1 & 2 & 5 \\
\hline 22. & LGG 705 & 9 & 3 & 5 & 1 & 2 & 9 & 1 & 1 & 1 & 2 & 2 & 5 & 3 & 2 & 9 & 2 & 3 & 2 & 1 & 3 & 3 & 2 & 2 & 5 \\
\hline 23. & LGG 706 & 9 & 5 & 5 & 1 & 2 & 9 & 1 & 1 & 2 & 2 & 2 & 5 & 3 & 2 & 9 & 1 & 3 & 2 & 3 & 3 & 2 & 2 & 2 & 5 \\
\hline 24. & LGG 707 & 9 & 5 & 5 & 1 & 2 & 9 & 9 & 3 & 1 & 2 & 2 & 5 & 3 & 2 & 9 & 1 & 3 & 2 & 1 & 5 & 2 & 1 & 1 & 5 \\
\hline 25. & LGG 708 & 9 & 5 & 5 & 1 & 2 & 9 & 1 & 2 & 1 & 2 & 2 & 3 & 3 & 2 & 9 & 2 & 3 & 2 & 1 & 3 & 3 & 2 & 2 & 5 \\
\hline 26. & LGG 709 & 9 & 3 & 5 & 1 & 2 & 9 & 1 & 3 & 2 & 2 & 2 & 5 & 3 & 2 & 9 & 1 & 3 & 2 & 1 & 3 & 4 & 2 & 2 & 5 \\
\hline 27. & LGG 710 & 1 & 3 & 5 & 1 & 2 & 9 & 1 & 2 & 2 & 2 & 2 & 5 & 3 & 2 & 9 & 2 & 3 & 2 & 1 & 3 & 2 & 1 & 1 & 5 \\
\hline 28. & LGG 711 & 9 & 3 & 5 & 1 & 2 & 9 & 1 & 1 & 2 & 2 & 2 & 3 & 3 & 2 & 9 & 1 & 3 & 2 & 3 & 3 & 2 & 1 & 1 & 5 \\
\hline 29. & LGG 712 & 1 & 5 & 5 & 1 & 2 & 9 & 1 & 1 & 2 & 2 & 2 & 5 & 3 & 2 & 9 & 1 & 3 & 2 & 1 & 3 & 3 & 2 & 2 & 5 \\
\hline 30. & LGG 460 & 9 & 3 & 5 & 1 & 2 & 9 & 1 & 2 & 1 & 2 & 2 & 5 & 3 & 2 & 9 & 1 & 3 & 2 & 1 & 3 & 2 & 1 & 1 & 5 \\
\hline
\end{tabular}

Anthocyanin colouration (1-Absent; 9-Present) Time of flowering (3-Early(<40 days); 5-Medium(40-50days); 7-Late( $>50$ days) Plant growth habit (3-Erect; 5-Semi erect; 7-Spreading) Plant habit

(1-Determinate; 3-Indeterminate) Stem colour (1-Green; 2-Green with purple splashes; 3-Purple) Stem pubescence (1-Absent; 9-Present), Leaflet lobes(1-Absent; 9-Present) Leaf shape (1-Deltoid; 2-Ovate;

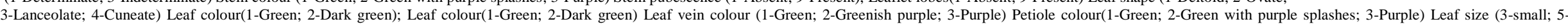

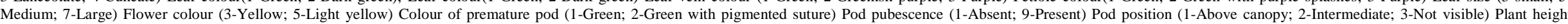

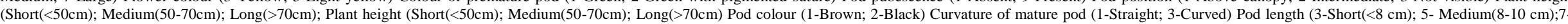
Long $(>10 \mathrm{~cm})$ ) Seed colour (1-Yellow; 2-Green; 3-Mottled; 4-Black) Seed luster (1-Shiny; 2-Dull) Seed shape (1-Oval; 3-Drum shape) Seed size (3-Small(<3 g); 5-Medium(3-5 g); 7-Large(>5 g)) 
Table.2 Grouping of elite improved lines based on DUS descriptor in Greengram

\begin{tabular}{|c|c|c|}
\hline Morphological characters & Character state & Genotypes \\
\hline \multirow{2}{*}{$\begin{array}{l}\text { Hypocotyl Anthocyanin } \\
\text { colouration }\end{array}$} & Absent & LGG684,LGG687,LGG689,LGG690, LGG698, LGG700, LGG701,LGG702, LGG703, LGG710,LGG712 \\
\hline & Present & $\begin{array}{l}\text { LGG685,LGG686,LGG688,LGG699,LGG692,LGG693,LGG694, LGG695,LGG696,LGG697, LGG699, LGG704, } \\
\text { LGG705, LGG706, LGG707, LGG708, LGG709, LGG711, LGG460 }\end{array}$ \\
\hline Plant growth habit & Semi-erect & All genotypes \\
\hline Plant habit & Determinate & All genotypes \\
\hline Petiole colour & $\begin{array}{l}\text { Green with purple } \\
\text { splashes }\end{array}$ & All genotypes \\
\hline \multirow[t]{2}{*}{ Plant height } & Short & $\begin{array}{l}\text { LGG684, LGG685, LGG686, LGG687, LGG688,LGG689, LGG690, LGG691, LGG692, LGG693,LGG694, LGG697, } \\
\text { LGG698, LGG699, LGG700,LGG701, LGG702, LGG703, LGG704, LGG705,LGG706, LGG707, LGG708, LGG709, } \\
\text { LGG710,LGG711, LGG712, LGG460 }\end{array}$ \\
\hline & Medium & LGG695, LGG696 \\
\hline Stem colour & $\begin{array}{l}\text { Green with purple } \\
\text { splashes }\end{array}$ & All genotypes \\
\hline Stem pubescence & Present & All genotypes \\
\hline \multirow[t]{2}{*}{ Leaflet lobes (terminal) } & Absent & $\begin{array}{l}\text { LGG685, LGG686, LGG687, LGG688, LGG689,LGG690, LGG691, LGG692, LGG693, LGG694,LGG695,LGG696, } \\
\text { LGG697, LGG699, LGG700,LGG702,LGG703,LGG704, } \\
\text { LGG705,LGG706,LGG708,LGG709,LGG710,LGG711,LGG712, LGG460 }\end{array}$ \\
\hline & Present & LGG684, LGG698, LGG701, LGG707 \\
\hline \multirow[t]{3}{*}{ Leaf shape (terminal) } & Deltoid & LGG684, LGG686, LGG687, LGG691, LGG693, LGG699, LGG700, LGG705, LGG706, LGG711,LGG712 \\
\hline & Ovate & $\begin{array}{l}\text { LGG685, LGG689, LGG690, LGG692, LGG694,LGG695, LGG696, LGG697, LGG698, LGG702,LGG703, LGG708, } \\
\text { LGG710, LGG460 }\end{array}$ \\
\hline & Lanceolate & LGG688, LGG701, LGG704, LGG707, LGG709 \\
\hline \multirow[t]{2}{*}{ Leaf colour } & Green & LGG688, LGG695, LGG700, LGG703, LGG704,LGG705, LGG707, LGG708, LGG460 \\
\hline & Dark green & $\begin{array}{l}\text { LGG684, LGG685, LGG686, LGG687, LGG689,LGG690, LGG691, LGG692, LGG693, LGG694,LGG696, LGG697, } \\
\text { LGG698, LGG699, LGG701,LGG702, LGG706, LGG709, LGG710, LGG711,LGG712 }\end{array}$ \\
\hline Leaf vein colour & Greenish purple & All genotypes \\
\hline \multirow[t]{3}{*}{ Leaf size } & Small & LGG708, LGG711 \\
\hline & Medium & $\begin{array}{l}\text { LGG684, LGG691, LGG692, LGG696, LGG697,LGG698, LGG701, LGG702, LGG703, LGG704,LGG705, LGG706, } \\
\text { LGG707, LGG709, LGG710,LGG712, LGG460 }\end{array}$ \\
\hline & Large & LGG685, LGG686, LGG687, LGG688, LGG689,LGG690, LGG693, LGG694, LGG695, LGG699,LGG700 \\
\hline Flower colour & Yellow & All genotypes \\
\hline Time of flowering & Early & LGG686, LGG687, LGG688, LGG689, LGG690, LGG691, LGG694, LGG695, LGG696, LGG700, LGG701, LGG703, \\
\hline
\end{tabular}




\begin{tabular}{|c|c|c|}
\hline & & LGG704, LGG705, LGG709, LGG710, LGG711, LGG712, LGG460 \\
\hline & Medium & LGG684, LGG685, LGG692, LGG693, LGG697,LGG698, LGG699, LGG702, LGG706, LGG707, LGG708, LGG712 \\
\hline \multirow[t]{2}{*}{ Colour of premature pod } & Green & LGG684, LGG685, LGG687, LGG689, LGG690 \\
\hline & $\begin{array}{l}\text { Green with pigmented } \\
\text { suture }\end{array}$ & $\begin{array}{l}\text { LGG686, LGG688, LGG691, LGG692, LGG693,LGG694, LGG695, LGG696, LGG697, LGG698,LGG699, LGG700, } \\
\text { LGG701, LGG702, LGG703,LGG704, LGG705, LGG706, LGG707, LGG708,LGG709, LGG710, LGG711, LGG712, } \\
\text { LGG460 }\end{array}$ \\
\hline \multirow[t]{2}{*}{ Pod pubescence } & Absent & LGG691, LGG692, LGG694, LGG697, LGG698 \\
\hline & Present & $\begin{array}{l}\text { LGG684, LGG685, LGG686, LGG687, LGG688,LGG689, LGG690, LGG692, LGG693, LGG695,LGG696, LGG699, } \\
\text { LGG700, LGG701, LGG702,LGG703, LGG704, LGG705, LGG706, LGG707,LGG708, LGG709, LGG710, LGG711, } \\
\text { LGG712,LGG460 }\end{array}$ \\
\hline \multirow[t]{3}{*}{ Pod position } & Above canopy & $\begin{array}{l}\text { LGG684, LGG686, LGG687, LGG688, LGG690,LGG691, LGG692, LGG694, LGG695, LGG696,LGG698, LGG700, } \\
\text { LGG701, LGG702, LGG706,LGG707, LGG709, LGG711, LGG712, LGG460 }\end{array}$ \\
\hline & Intermediate & LGG685, LGG689, LGG693, LGG704, LGG705,LGG708, LGG710 \\
\hline & Not visible & LGG697, LGG699, LGG703 \\
\hline Pod colour & Black & All genotypes \\
\hline \multirow[t]{2}{*}{ Curvature of mature pod } & Straight & $\begin{array}{l}\text { LGG684, LGG685, LGG686, LGG687, LGG688,LGG689, LGG690, LGG691, LGG692, LGG693,LGG694, LGG695, } \\
\text { LGG698, LGG700, LGG701,LGG703, LGG704, LGG705, LGG707, LGG708,LGG709, LGG710, LGG712, LGG460 }\end{array}$ \\
\hline & Curved & LGG696, LGG697, LGG699, LGG702, LGG706,LGG711 \\
\hline \multirow[t]{2}{*}{ Pod length } & Short & $\begin{array}{l}\text { LGG684, LGG685, LGG686, LGG687, LGG688,LGG689, LGG691, LGG692, LGG693, LGG694,LGG695, LGG696, } \\
\text { LGG697, LGG699, LGG701,LGG703, LGG705, LGG706, LGG708, LGG709,LGG710, LGG711, LGG712, LGG460 }\end{array}$ \\
\hline & Medium & LGG690, LGG698, LGG700, LGG702, LGG704,LGG707 \\
\hline \multirow[t]{4}{*}{ Seed colour } & Yellow & $\begin{array}{l}\text { LGG686, LGG687, LGG688, LGG689, LGG690,LGG691, LGG692, LGG693, LGG694, LGG695,LGG696, LGG697, } \\
\text { LGG698, LGG699 }\end{array}$ \\
\hline & Green & LGG685, LGG701, LGG702, LGG703, LGG704,LGG706, LGG707, LGG710, LGG711, LGG460 \\
\hline & Mottled & LGG684, LGG686, LGG700, LGG705, LGG707,LGG712 \\
\hline & Black & LGG709 \\
\hline \multirow[t]{2}{*}{ Seed luster } & Shiny & $\begin{array}{l}\text { LGG684, LGG685, LGG686, LGG687, LGG690,LGG691, LGG693, LGG694, LGG695, LGG696,LGG698, LGG700, } \\
\text { LGG702, LGG703, LGG704,LGG707, LGG710, LGG711, LGG460 }\end{array}$ \\
\hline & Dull & LGG688, LGG689, LGG692, LGG699, LGG701,LGG705, LGG706, LGG708, LGG709, LGG712 \\
\hline \multirow[t]{2}{*}{ Seed shape } & Oval & $\begin{array}{l}\text { LGG684, LGG685, LGG686, LGG687, LGG689,LGG690, LGG694, LGG695, LGG696, LGG702,LGG703, LGG707, } \\
\text { LGG710, LGG711, LGG460 }\end{array}$ \\
\hline & Drum shaped & $\begin{array}{l}\text { LGG688, LGG691, LGG692, LGG693, LGG697,LGG698, LGG699, LGG700, LGG701, LGG704,LGG705, LGG706, } \\
\text { LGG708, LGG709, LGG712 }\end{array}$ \\
\hline Seed Size & Medium & All genotypes \\
\hline
\end{tabular}


Table.3 DUS descriptors of Elite improved Greengram lines developed at RARS, Lam, Guntur, Andhra Pradesh

\begin{tabular}{|c|c|c|c|}
\hline S.No. & $\begin{array}{l}\text { Name of } \\
\text { genotype }\end{array}$ & $\begin{array}{l}\text { No. of } \\
\text { traits }\end{array}$ & Specific traits \\
\hline 1. & LGG 684 & 15 & $\begin{array}{l}\text { Medium maturity, short plant height, presence of leaflet lobes, deltoid leaf shape, dark green coloured leaves, medium leaf size, green coloured premature pod, pod } \\
\text { position was above canopy, straight curvature of pod, presence of pod pubescence, short pod length, black coloured mature pod, mottled seed colour, shiny seed } \\
\text { luster, oval shaped seed. }\end{array}$ \\
\hline 2. & LGG 685 & 14 & $\begin{array}{l}\text { Anthocyanin colouration, medium maturity, short plant height, ovate leaf shape, dark green leaf colour, large leaf size, green colour premature pod, presence of pod } \\
\text { pubescence, intermediate pod position, straight curvature of pod, short pod length, green coloured seed, shiny seed luster, oval shaped seed. }\end{array}$ \\
\hline 3. & LGG 686 & 13 & $\begin{array}{l}\text { Anthocyanin colouration, early maturity, deltoid leaf shape, dark green leaf colour, large leaf size, green with pigmented suture coloured pod, presence of pod } \\
\text { pubescence, pod position was above canopy, straight curvature of pod, short pod length, mottled seed colour, shiny seed luster, oval shaped seed. }\end{array}$ \\
\hline 4. & LGG 687 & 12 & $\begin{array}{l}\text { Early maturity, deltoid leaf shape, dark green leaf colour, large leaf size, green coloured premature pod, presence of pod pubescence, pod position was above } \\
\text { canopy, straight curvature of pod, short pod length, yellow seed colour, shiny seed luster, oval shaped seed. }\end{array}$ \\
\hline 5. & LGG 688 & 13 & $\begin{array}{l}\text { Anthocyanin colouration, short plant height, lanceolate leaf shape, green leaf colour, large leaf size, green with pigmented suture coloured pod, presence of pod } \\
\text { pubescence, pod position was above canopy, straight curvature of pod, short pod length, yellow seed colour, dull seed luster, drum shaped seed. }\end{array}$ \\
\hline 6. & LGG 689 & 13 & $\begin{array}{l}\text { Anthocyanin colouration, short plant height, ovate leaf shape, dark green leaf colour, large leaf size, green coloured premature pod, presence of pod pubescence, } \\
\text { intermediate pod position, straight curvature of pod, short pod length, yellow seed colour, dull seed luster, oval shaped seed. }\end{array}$ \\
\hline 7. & LGG 690 & 13 & $\begin{array}{l}\text { Early maturity, short plant height, ovate leaf shape, dark green leaf colour, large leaf size, green coloured premature pod, presence of pod pubescence, pod position } \\
\text { was above canopy, straight curvature of pod, medium pod length, yellow seed colour, shiny seed luster, oval shaped seed. }\end{array}$ \\
\hline 8. & LGG 691 & 13 & $\begin{array}{l}\text { Early maturity, short plant height, deltoid leaf shape, dark green leaf colour, medium leaf size, green with pigmented suture coloured pod, pod pubescence absent, } \\
\text { pod position was above canopy, straight curvature of pod, short pod length, yellow seed colour, shiny seed luster, drum shaped seed. }\end{array}$ \\
\hline 9. & LGG 692 & 14 & $\begin{array}{l}\text { Anthocyanin colouration, medium maturity, short plant height, ovate leaf shape, dark green leaf colour, medium leaf size, green with pigmented suture coloured } \\
\text { pod, pod pubescence absent, pod position was above canopy, straight curvature of pod, short pod length, yellow seed colour, dull seed luster, drum shaped seed. }\end{array}$ \\
\hline 10. & LGG 693 & 14 & $\begin{array}{l}\text { Anthocyanin colouration, medium maturity, short plant height, deltoid leaf shape, dark green leaf colour, large leaf size, green with pigmented suture coloured pod, } \\
\text { presence of pod pubescence, intermediate pod position, straight curvature of pod, short pod length, yellow seed colour, shiny seed luster, drum shaped seed. }\end{array}$ \\
\hline 11. & LGG 694 & 14 & $\begin{array}{l}\text { Anthocyanin colouration, early maturity, short plant height, ovate leaf shape, dark green leaf colour, large leaf size, green with pigmented suture coloured pod, pod } \\
\text { pubescence absent, pod position was above canopy, straight curvature of pod, short pod length, yellow seed colour, shiny seed luster, oval shaped seed }\end{array}$ \\
\hline 12. & LGG 695 & 14 & $\begin{array}{l}\text { Anthocyanin colouration, early maturity, medium plant height, ovate leaf shape, green leaf colour, large leaf size, green with pigmented suture coloured pod, } \\
\text { presence of pod pubescence, pod position was above canopy, straight curvature of pod, short pod length, yellow seed colour, shiny seed luster, oval shaped seed }\end{array}$ \\
\hline 13. & LGG 696 & 14 & $\begin{array}{l}\text { Anthocyanin colouration, early maturity, medium plant height, ovate leaf shape, dark green leaf colour, medium leaf size, green with pigmented suture coloured } \\
\text { pod, presence of pod pubescence, pod position was above canopy, curved curvature of pod, short pod length, yellow seed colour, shiny seed luster, oval shaped } \\
\text { seed. }\end{array}$ \\
\hline 14. & LGG 697 & 13 & $\begin{array}{l}\text { Anthocyanin colouration, medium maturity, short plant height, ovate leaf shape, dark green leaf colour, green with pigmented suture coloured pod, pod pubescence } \\
\text { absent, podposition was not visible, curved curvature of pod, short pod length, yellow seed colour, shiny seed luster, drum shaped seed. }\end{array}$ \\
\hline 15. & LGG 698 & 14 & $\begin{array}{l}\text { Medium maturity, short plant height, presence of leaflet lobes, ovate leaf shape, dark green leaf colour, medium leaf size, green with pigmented suture coloured } \\
\text { pod, pod pubescence absent, pod position was above canopy, straight curvature of pod, medium pod length, yellow seed colour, shiny seed luster, drum shaped } \\
\text { seed. }\end{array}$ \\
\hline 16. & LGG 699 & 14 & $\begin{array}{l}\text { Anthocyanin colouration, medium maturity, short plant height, pod position was not visible, deltoid leaf shape, large leaf size, green with pigmented suture coloured } \\
\text { pod, presence of pod pubescence, podposition was not visible,curved curvature of pod, short pod length, yellow seed colour, dull seed luster, drum shaped seed. }\end{array}$ \\
\hline 17. & LGG 700 & 13 & Early maturity, short plant height, deltoid leaf shape, green leaf colour, large leaf size, green with pigmented suture coloured pod, presence of pod pubescence, pod \\
\hline
\end{tabular}




\begin{tabular}{|c|c|c|c|}
\hline & & & position was above canopy, straight curvature of pod, medium pod length, mottled seed colour, shiny seed luster, drum shaped seed. \\
\hline 18. & LGG 701 & 14 & $\begin{array}{l}\text { Early maturity, short plant height, presence of leaflet lobes, lanceolate leaf shape, dark green leaf colour, medium leaf size, green with pigmented suture coloured } \\
\text { pod, presence of pod pubescence, pod position was above canopy, straight curvature of pod, short pod length, green coloured seed, dull seed luster, drum shaped } \\
\text { seed. }\end{array}$ \\
\hline 19. & LGG 702 & 13 & $\begin{array}{l}\text { Early maturity, short plant height, ovate leaf shape, dark green leaf colour, medium leaf size, green with pigmented suture coloured pod, presence of pod } \\
\text { pubescence, pod position was above canopy, curved curvature of pod, medium pod length, green coloured seed, shiny seed luster, oval shaped seed. }\end{array}$ \\
\hline 20. & LGG 703 & 13 & $\begin{array}{l}\text { Early maturity, short plant height, ovate leaf shape, green leaf colour, medium leaf size, green with pigmented suture coloured pod, presence of pod pubescence, } \\
\text { pod position was not visible, straight curvature of pod, short pod length, green coloured seed, shiny seed luster, oval shaped seed. }\end{array}$ \\
\hline 21. & LGG 704 & 14 & $\begin{array}{l}\text { Anthocyanin colouration, early maturity, short plant height, lanceolate leaf shape, green leaf colour, medium leaf size, green with pigmented suture coloured pod, } \\
\text { presence of pod pubescence, intermediate pod position, straight curvature of pod, medium pod length, green coloured seed, shiny seed luster, drum shaped seed. }\end{array}$ \\
\hline 22. & LGG 705 & 14 & $\begin{array}{l}\text { Anthocyanin colouration, early maturity, short plant height, deltoid leaf shape, green leaf colour, medium leaf size, green with pigmented suture coloured pod, } \\
\text { presence of pod pubescence, intermediate pod position, straight curvature of pod, short pod length, mottled seed colour, dull seed luster, drum shaped seed. }\end{array}$ \\
\hline 23. & LGG 706 & 14 & $\begin{array}{l}\text { Anthocyanin colouration, medium maturity, short plant height, deltoid leaf shape, dark green leaf colour, medium leaf size, green with pigmented suture coloured } \\
\text { pod, presence of pod pubescence, pod position was above canopy, curved curvature of pod, short pod length, green coloured seed, dull seed luster, drum shaped } \\
\text { seed. }\end{array}$ \\
\hline 24. & LGG 707 & 15 & $\begin{array}{l}\text { Anthocyanin colouration, medium maturity, short plant height, presence of leaflet lobes, lanceolate leaf shape, green leaf colour, medium leaf size, green with } \\
\text { pigmented suture coloured pod, presence of pod pubescence, straight curvature of pod, medium pod length, green coloured seed, mottled seed colour, shiny seed } \\
\text { luster, oval shaped seed. }\end{array}$ \\
\hline 25. & LGG 708 & 14 & $\begin{array}{l}\text { Anthocyanin colouration, medium maturity, short plant height, ovate leaf shape, green leaf colour, small leaf size, green with pigmented suture coloured pod, } \\
\text { presence of pod pubescence, pod position was above canopy, intermediate pod position, straight curvature of pod, short pod length, dull seed luster, drum shaped } \\
\text { seed. }\end{array}$ \\
\hline 26. & LGG 709 & 13 & $\begin{array}{l}\text { Early maturity, short plant height, lanceolate leaf shape, dark green leaf colour, medium leaf size, green with pigmented suture coloured pod, presence of pod } \\
\text { pubescence, pod position was above canopy, straight curvature of pod, short pod length, black seed colour, dull seed luster, drum shaped seed. }\end{array}$ \\
\hline 27. & LGG 710 & 13 & $\begin{array}{l}\text { Early maturity, short plant height, ovate leaf shape, medium leaf size, dark green leaf colour, presence of pod pubescence, green with pigmented suture coloured } \\
\text { pod, intermediate pod position, straight curvature of pod, short pod length, green coloured seed, shiny seed luster, oval shaped seed. }\end{array}$ \\
\hline 28. & LGG 711 & 14 & $\begin{array}{l}\text { Anthocyanin colouration, early maturity, short plant height, deltoid leaf shape, dark green leaf colour, small leaf size, green with pigmented suture coloured pod, } \\
\text { presence of pod pubescence, pod position was above canopy, curved curvature of pod, short pod length, shiny seed luster, green seed colour, oval shaped seed. }\end{array}$ \\
\hline 29. & LGG 712 & 13 & $\begin{array}{l}\text { Early maturity, short plant height, deltoid leaf shape, dark green leaf colour, medium leaf size, green with pigmented suture coloured pod, presence of pod } \\
\text { pubescence, pod position was above canopy, straight curvature of pod, short pod length, mottled seed colour, dull seed luster, drum shaped seed. }\end{array}$ \\
\hline 30. & LGG 460 & 14 & $\begin{array}{l}\text { Anthocyanin colouration, medium maturity, short plant height, ovate leaf shape, green leaf colour, medium leaf size, green with pigmented suture coloured pod, } \\
\text { presence of pod pubescence, pod position was above canopy, straight curvature of pod, short pod length, green coloured seed, shiny seed luster, oval shaped seed. }\end{array}$ \\
\hline
\end{tabular}




\section{Seed characters}

The price of premium genotypes of greengram or consumer acceptance of a variety is decided by the seed characteristics like colour, size and shape (Pratap et al., 2018). Varieties with oval shining green grains with medium size are preferred over dull/ brown/ black and drum shaped grains. Seed morphological characters like colour, luster, size and shape were observed at mature seed stage of plant in the present experiment (Table-2).

All these characters were grouped into distinct groups. Seed colour was yellow in fourteen genotypes, green in ten genotypes, mottled in five genotypes and black in one genotype (LGG 709), respectively. Seed color determines phytic acid levels in the seed and there are reports that yellow seeded genotypes had low phytic acid content which can be used as a donor for quality improvement of greengram seeds (Tajoddin et al., 2011).

Seed luster was shiny in 19 genotypes and dull in 11 genotypes. Seed shape was oval in 15 genotypes and drum shaped in 15 genotypes. Medium seed size was noted in all the genotypes. Thus, seed morphological traits form very good markers for the purity testing and identification except seed size. Some of the lines are having consumer accepted seed traits for fetching premium price in the market. Venkateswarlu (2001) and Khajudparn and Tantasawat (2011) also discussed the usefulness of seed characters in the characterization of lines in greengram.

Among the 24 morphological DUS traits observed one character (seed colour) showed tetramorphic variation, four characters (leaf shape, leaf size, pod position and seed size) recorded trimorphic variation; eleven characters (anthocyanin colouration of cotyledons, plant height, leaflet lobes, leaf colour, time of flowering, colour of premature pod, pod pubescence, pod length, curvature of the pod, seed luster and seed shape) indicated the dimorphic grouping and plant habit, growth habit, petiole colour, stem colour and pubescence, leaf vein colour, flower colour, pod colour and seed size showed no variation i.e., monomorphic grouping indicating the existence of remarkable amount of genetic variability in these genotypes which have great potential to assign distinctive morphological profiles from combination of morphological DUS traits which could be used for elite improved lines identification and characterization as well as selection of diverse parents in hybridization programme for more heterotic response and generation of better segregants in munbean breeding (Table-3). Verma et al., (2017) also exploited DUS characterization for the identification during hybrid seed production in rice for the identification of off types.

In the present investigation, stem pigmentation and flower colour are same in all the lines and are not useful for discrimination but anthocyanin colouration at cotyledanory stage, plant, leaf, pod and seed characteristics are having lot of variability which can be exploited for the elite lines identification and utilization as reported by Patel et al., (2019) and facilitate the easy registration with these distinct characters present in the genotypes with PPVFRA.

Further, the study also highlighted the importance of incorporation of new material in the breeding programmes to widen the genetic base of the crop at the Research Station. Thus, characterization of elite improved lines forms a great significance in the identification of lines, registration of lines with PPV \&FRA and maintenance of lines along with the information on the genetic base of the breeding material at the Research Station. 


\section{Acknowledgement}

First author is highly thankful to Acharya NG Ranga Agricultural University, Lam, Guntur for providing the stipend during the M. Sc (Ag.) programme.

\section{References}

Abbas G, Asghar M Jand ShahT M 2010 Genetic diversity in mungbean (Vigna radiata L.) germplasm. Pak. J. Bot., 42: 3845-3495.

Jain S K, Khare D,Bhale M S and Raut N D 2002Characterization of mung bean varieties for verification of genetic purity. Seed Tech News, 32: 200-201.

Janghel D K, Krishan Kumar, Sunil Rand ChhabraA K 2020 Genetic diversity analysis, characterization and evaluation of elite chickpea (Cicer arietinum L.) genotypes. Int.J. Curr. Microbiol. App. Sci., 9: 199-209.

Katiyar P K, Dixit G Pand SinghB B2008 Morphological characterization of greengram (Vigna radiata) varieties and their application for distinctness, uniformity and stability testing. Indian J. Agri. Sci.,78: 439-444.

Katiyar P K, Dixit GP and SinghB B 2007 Ancestral relationship of greengram (Vigna radiata) advanced breeding lines developed in India. Indian J. Agri. Sci.,77: 579-582.

Kaur R, ToorAK, Geeta Bassi and BainsT S 2017 Characterization of mungbean (Vigna radiata L. Wilczek) varieties using morphological and molecular descriptors. Int.J. Curr.Microbiol. App. Sci., 6: 1609-1618.

Khajudparn P and TantasawatP 2011 Relationships and variability of agronomic and physiological characters in mungbean. African J. Biotechnol., 10: 9992-10000.

Khattak GSS, HaqM A, Ashraf $M$ and
SaleemM 2000 Inheritance of hypocotyl colour and pubescence in mungbean (Vigna radiata (L.) Wilczek). J. Sci. Ind. Res., Iran, 11: 79-81.

Lee Y S, LeeJ Y, Kim D K,YoonC Y,BakG C,ParkI J, BangG P, Moon J K,Oh Y J and KminK S 2004A new high-yielding mungbean cultivar, "Samgang" with lobed leaflet. Korean J. Breed. Sci., 36: 183-184.

Mukherjee A and Pradhan K. 2002. Genetics of mungbean: I. Anthocyanin pigment in hypocotyl. J.Interacademicia., 6: 434437.

Patel J D, Patel J Band Chetariya C P 2019 Characterization of mungbean (Vigna radiata (L.) Wilczek) genotypes based on plant morphology. Indian J. Pure and App. Biosci., 7: 433-443.

Piyada T, JuthamasT, ThongchaiP, Thanawit T, Chutamas P, WorapaSand ThitipornM 2010 Variety identification and genetic relationships of mungbean and blackgram in Thailand based on morphological characters and ISSR analysis. African J. Biotechnol., 9: 44524464.

Pratap A, GuptaDS, Singh BBand KumarS 2013 Development of super early genotypes in greengram [Vigna radiata (L.) Wilczek]. Legume Res.,36: 105110.

PratapA, Nupur Malviya, Sanjeev Gupta, Rakhi Tomar, Vankat Raman Pandey and Umashanker Prajapati 2018 Field characterization of endemic wild Vigna accessions collected from biodiversity hotspots of India to identify promising genotypes for multiple agronomic and adaptive traits. Legume Res., 41: 490499.

Protection of Plant Varieties and Farmers' Rights Authority (PPV \& FRA), 2007.

Ramakrishnan M N, Ray-Yu Yang, WarwickJ E, DilThavarajah, Pushparajah T, Jacqueline d'A Hand Keatinge J D H 
2013 Biofortification of mungbean (Vigna radiata) as a whole food to enhance human health. J. Sci. Food Agric., 93: 1805-1813.

Sunil N, Someswara RaoP, Natarajan S, Jairam Reddy, Chakrabarty SK, Ashok J and BhistIS 2014 Diversity in the landraces of greengram (Vigna radiata (L) R. Wilczek) collected from Tribal communities of Peninsular India. Photon, 114: 392-400.

Sunil N, Sivaraj N and Chakrabarty, S K 2013 Characterization and evaluation of mungbean (Vigna radiata L. Wilczek) germplasm from Andhra Pradesh.
Indian J. PGR.,16: 18-20.

Tajoddin M, ShindeMand LalithaJ 2011. Phytic acid and mineral content of mungbean cultivars. J.Food Legumes, 24: 163-164.

Venkateshwarlu O. 2001. Correlation and path analysis in greengram. Legume Res., 24: 115-117.

Verma R. L, Singh S., Singh P., Kumar V., Singh, S.P., Singh S., Samantaray S. and Singh. 2017. Genetic purity assessment of indica rice hybrids through DNA fingerprinting and grow out test. Journal of Environ. Biol., 38: 1321-1331.

\section{How to cite this article:}

Sheena Sabatina, A., M. Lal Ahamed, J. V. Ramana and Harisatyanarayana, N. 2021. DUS Characterization of Elite Improved Lines of Greengram [Vigna radiata (L.) Wilczek]. Int.J.Curr.Microbiol.App.Sci. 10(01): 3380-3391. doi: https://doi.org/10.20546/ijcmas.2021.1001.398 